\title{
LA INVESTIGACIÓN EN LA ZONA CENTRO BOGOTÁ CUNDINAMARCA: UN ESCENARIO PARA LA CONSTRUCCIÓN DE CONOCIMIENTO
}

\author{
Juan Carlos Hernández Barrero, ${ }^{67}$ \\ Sonia Bibiana Rojas Wilches ${ }^{68}$
}

\section{INTRODUCCIÓN}

\begin{abstract}
En este artículo se busca visibilizar los avances significativos, que en materia de investigación, ha tenido la Zona Centro Bogotá Cundinamarca (ZCBC), producto del esfuerzo de estudiantes, tutores, líderes locales de investigación y la coordinación zonal de investigación, integrados en un ambiente de aprendizaje colectivo y colaborativo, dentro de un sistema fractal y reticular para la producción de conocimiento.
\end{abstract}

Para ello, se hace una identificación de la zona, a partir del acercamiento a cada uno de los centros que la integran, su contexto y desarrollo en términos de investigación. Luego, el análisis se enfoca en el impacto que han tenido algunas estrategias para la consolidación de una cultura investigativa, habilitando la participación de grupos y semilleros locales, en escenarios nacionales de promoción y difusión del conocimiento, como la Convocatoria abierta por el Sistema de Investigación Unadista (SIUNAD) para proyectos de investigación en el 2009, y el IV Encuentro Nacional de Investigación; el balance de dicha participación se desarrolla como conclusión de este artículo.

Recibido: 10 de julio de 2009

Aceptado: 23 de octubre de 2009

\section{Una zona con proyección}

Iniciando el año 2009 se genera un proceso de reestructuración en la UNAD en cuanto a su cubrimiento geográfico nacional, dando paso a la configuración de la Zona Centro Bogotá Cundinamarca integrada por 6 Centros de Educación a Distancia (CEAD). A continuación, se presenta una breve identificación del departamento y los municipios que la componen, para luego pasar al análisis de los avances que ha tenido la investigación en éste ámbito regional.

El departamento de Cundinamarca se sitúa en el centro del país, sobre la Cordillera Oriental. Posee una población mestiza en su mayoría, producto de la mezcla de los colonos españoles con los indígenas que poblaban el territorio. Su economía se concentra principalmente en el sector agropecuario, seguido por la industria, los servicios y el comercio. Desde la Colonia, este departamento, ha sido considerado foco económico, político y cultural de Colombia.

67 Coordinador Zonal de Investigación - Zona Centro Bogotá Cundinamarca

68 Líder de Investigación CEAD Zipaquirá 
Actualmente, el departamento se encuentra dividido en 15 provincias: Almeidas, Alto Magdalena, Bajo Magdalena, Gualivá, Guavio, Magdalena Centro, Medina, Oriente, Rionegro, Sabana Centro, Sabana Occidente, Soacha, Sumapaz, Tequendama y Ubaté, en donde se asientan 116 municipios y el Distrito Capital.

La UNAD actúa en gran parte de estas provincias a través de los CEAD que se encuentran ubicados en 5 municipios (CEAD Gachetá, CEAD Arbeláez, CEAD Girardot, CEAD Facatativá y CEAD Zipaquirá) y en la ciudad de Bogotá con el Nodo Zonal José Acevedo y Gómez.

Acorde con la misión institucional, estos centros han venido desarrollando procesos investigativos, que aunados a la proyección social y la acción pedagógica, han permitido incidir en las dinámicas del desarrollo social de la región. En ese contexto, la ZCBC cuenta con actores en el proceso investigativo, quienes con interés se han venido incorporando al sIUNAD.

A continuación se muestra una caracterización específica por cada centro.

\section{Gachetá:}

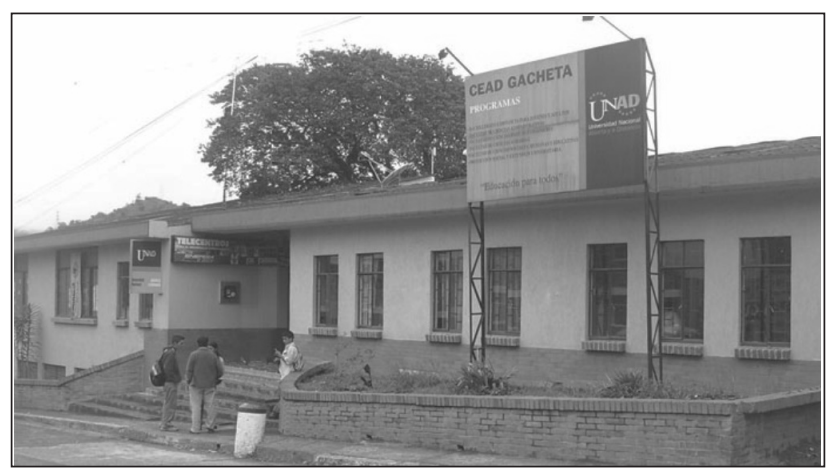

Figura 1. CEAD Gachetá

El municipio de Gachetá es la capital de la provincia del Guavio, se localiza en el nororiente del departamento de Cundinamarca, a 99 kilómetros del Distrito Capital, sobre la vía que de Bogotá conduce a Ubalá y Gachalá. Allí nace en 1985 el CEAD que lleva su nombre, el cual atiende en la actualidad alrededor de 126 estudiantes en pregrado y 164 en Bachillerato, provenientes de diferentes municipios, tanto de la región, como de la provincia de Medina.

Es un CEAD naciente en investigación, se encuentra organizando su equipo de trabajo. En el periodo I de 2009 inicia su participación en las actividades del Sistema de Investigación Unadista de la zona. En este momento sus actores no hacen presencia en grupos ni semilleros de investigación, manifiestan estar en proceso de consolidación. 


\section{Arbeláez:}

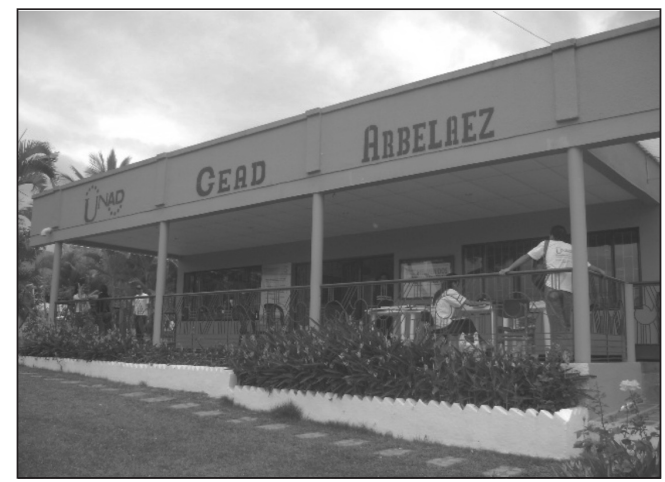

Figura 2. CEAD Arbeláez

Arbeláez se encuentra ubicado en la provincia de Sumapaz. Conocido como la "Ciudad tranquila y acogedora de Colombia", este municipio posee una temperatura cuyo promedio anual es de $20^{\circ} \mathrm{C}$. Sus principales actividades económicas son la agricultura, la ganadería y el comercio. Tiene una población de 23.420 habitantes.

El CEAD Arbeláez fue fundado en Julio de 1986. Hoy en día atiende a un número aproximado de 600 estudiantes. Su fortaleza está dada por la participación de sus investigadores en proyectos de investigación aprobados en convocatorias externas; es el caso de la convocatoria de Colciencias, que ha motivado la integración de nuevos grupos y el avance de nuevas propuestas de investigación.

\section{Girardot:}

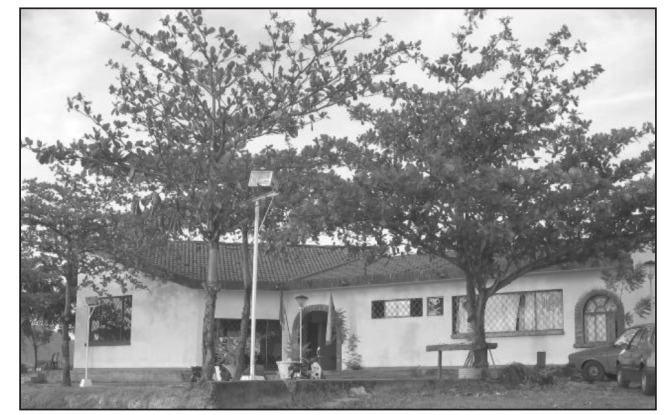

Figura 3. CEAD Girardot

Es uno de los municipios más grandes del departamento. Situado en el extremo sur-oriente de Cundinamarca al margen derecho del Río Magdalena y a una altura aproximada de $288 \mathrm{~m}$. sobre el nivel del mar; su actividad económica está centrada en el comercio, resultado de la importante dinámica turística, generada por su agradable clima y su estratégica ubicación. En esta ciudad, se encuentra ubicado el CEAD Girardot, en donde se vienen consolidando dos 
grupos de investigación: SCIRE, con participación de las escuelas de Ciencias de la Educación y Ciencias Sociales Artes y Humanidades; y GIREG, con participación de las escuelas Ciencias Sociales Artes y Humanidades, Ciencias Básicas Tecnología E Ingeniería y Ciencias Administrativas Contables Económicas y de Negocios.

\section{Facatativá:}

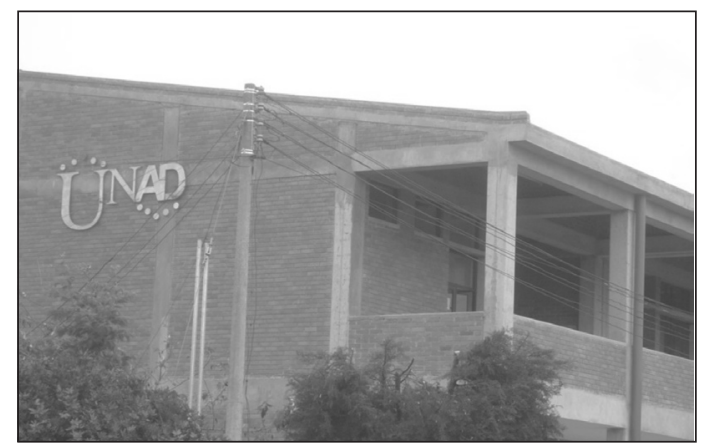

Figura 4. CEAD Facatativá

Facatativá es la capital de la provincia Sabana Occidente y es parte del área metropolitana de Bogotá. Entre los lugares más atractivos de este municipio se encuentra el Parque Arqueológico Piedras del Tunjo, el cual es comúnmente llamado "Piedras de Tunja" donde se encuentran petroglifos. Su economía es esencialmente agrícola y ganadera, se resalta el cultivo de hortalizas, frutas y flores; así mismo, posee un importante sector industrial y diversas empresas prestadoras de servicios.

La participación del CEAD Facatativá es muy representativa en términos de los semilleros de investigación. A pesar de no contar con grupos de investigación estos semilleros buscan ser apadrinados por grupos de otros centros. Las proyecciones del CEAD están en la dinámica de consolidar un equipo de investigación con los tutores que vienen trabajando y generar otros escenarios en materia de formación en esta área.

\section{Zipaquirá:}

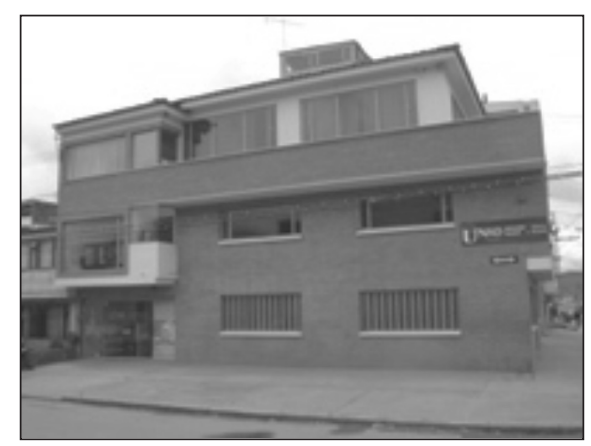

Figura 5. CEAD Zipaquirá 
Reconocido por su Catedral de Sal, este municipio ha sido bautizado "La Capital Salinera de Colombia", pues su actividad económica se centra justamente en la explotación minera de este producto, así como en el comercio y el turismo. La UNAD está presente allí desde hace 26 años, y ha logrado impactar diferentes provincias del departamento a través de los Centros Comunitarios de Educación Virtual (CAV), que se encuentran adscritos al CEAD Zipaquirá (CAV Tocancipá, CAV Ubaté y CAV Pacho).

El área de investigación se viene consolidando en este CEAD desde el año 2007, tiempo en el cual se ha logrado el registro de 10 semilleros de investigación en el sIUNAD, pertenecientes a diferentes Escuelas. Asimismo, el aval del Grupo de Investigación Chicaquicha, de la Escuela de Ciencias Sociales Artes y Humanidades, que viene desarrollando un proyecto financiado por SIUNAD ganador en la convocatoria 2009. Dos grupos de investigación más, el Grupo G’Kaira y el Grupo Producción Agropecuaria Sostenible, buscan el aval de sIUNAD, para participar en la convocatoria de proyectos de investigación 2010.

\section{José Acevedo y Gómez:}

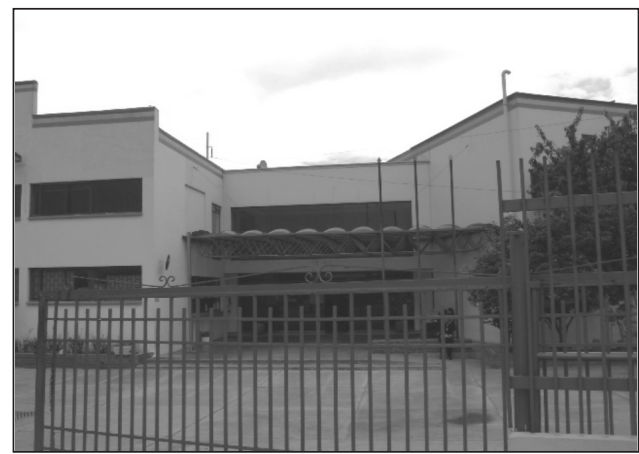

Figura 6. NODO ZONAL José Acevedo y Gómez

Bogotá es el principal centro urbano, económico, político, social y cultural del país. Su economía depende principalmente de los servicios y de la industria. La Constitución de 1991 le dio el carácter de Distrito Capital. Es la ciudad más poblada de Colombia, cuenta en la actualidad con un total aproximado de 6.776.009 habitantes.

Ubicado en esta ciudad se encuentra el Nodo Zonal José Acevedo y Gómez. Es el centro más grande de la zona y del país, su participación en los procesos investigativos es muy consecuente con el número de actores que lo componen, es un centro que aporta de manera significativa a la investigación y a la Universidad. Varios de sus actores están vinculados a los grupos de investigación que fueron clasificados en la pasada convocatoria de Colciencias, en este sentido, se percibe que la vinculación de investigadores a los grupos de investigación más fuertes en su consolidación, habilita un escenario de actuación de gran solidez y resultados satisfactorios en las convocatorias de investigación. 


\section{Algunas experiencias y estrategias significativas}

La reciente configuración de la $\mathrm{ZCBC}$, ha generado un proceso de reorganización que afecta su sistema de investigación, por lo que surge la necesidad de implementar diversas estrategias para la articulación de los centros, dado que la concentración de los procesos de gestión en el nodo zonal, venía originando una menor visibilidad y desarrollo de los centros más pequeños, restándoles reconocimiento y oportunidades de avance en sus procesos investigativos.

La primera acción encaminada a la consecución de este objetivo fue la identificación de unos líderes de investigación por cada centro, como estrategia para la consolidación de un equipo base para el trabajo investigativo en la zona, habilitando canales de comunicación entre sus diferentes actores efectivos para el desarrollo de los principios de fractalidad y reticularidad, propios de la organización sistémica de la UNAD.

En ese sentido, se planteó la realización de un primer encuentro zonal de investigación, en el mes de marzo, a través del desarrollo de un workshop (taller), que permitió fundamentar un diagnóstico participativo para el reconocimiento inicial de los procesos que se venían desarrollando y la identificación de necesidades específicas en investigación desde lo local.

Asimismo, se consolida la estrategia de conversatorios en investigación en los diferentes CEAD de la zona, en donde se realiza un trabajo con estudiantes y tutores sobre el proceso de conformación de grupos y semilleros de investigación, la organización y funcionamiento del SIUNAD, y el procedimiento para la presentación de proyectos de investigación a las convocatorias.

La difusión de estos procesos, así como su seguimiento y retroalimentación, se logra a través de la apertura del sitio web de investigación de la zona, creado como un espacio de discusión, gestión y visibilización de los diferentes actores que se encuentran vinculados a los grupos y semilleros, y de la producción de conocimiento que desde estos espacios se viene desarrollando.

Tales estrategias han dado como resultado el fomento de una cultura investigativa que se compone, entre otros, de elementos como la organización, las normas, los valores, y la formación en investigación, elementos que permiten interiorizar y poner en práctica la investigación como un componente escencial para el desarrollo personal, profesional y social.

La organización implica la integración de grupos y semilleros alrededor de unas líneas de interés, que han sido identificadas en la zona y que son pertinentes para las problemáticas y necesidades regionales, con lo que se logra el fortalecimiento del sistema de investigación y la consolidación de redes de conocimiento.

Las normas se generan desde la aplicación de unos estándares reconocidos por la comunidad científica para el desarrollo de la investigación, estos estándares se evidencian en los procesos y procedimientos establecidos desde el sistema de investigación como parte del proceso de gestión de calidad; los talleres realizados en la zona han jugado un papel funadmental en el conocimiento y puesta en práctica de dichos estándares, lo que ha permitido la participación efectiva de grupos y semilleros en convocatorias internas de la Universidad. 
Por su parte, los valores permiten el posicionamiento y permanencia de la cultura investigativa como elemento fundamental del quehacer pedagógico en la Universidad, estos valores se fomentan a partir del trabajo en equipo, la participación de tutores y estudiantes en eventos académicos para intercambiar experiencias, etc.

Por último, el campo de la investigación formativa está referenciado desde el trabajo en los semilleros. Se entiende el semillero como un escenario en donde los estudiantes interactúan entre sí, y con profesionales que los asesoran, lo que les permite la adquisición de competencias investigativas.

\section{Desarrollos investigativos}

La participación de la zona en la convocatoria de investigación es muy representativa: se presentan 12 proyectos de semilleros de los 35 registrados a nivel nacional, equivalentes al 34,3\%. En grupos de investigación se postulan 38 proyectos de los 107 a nivel nacional, equivalentes al 35,5\% de los proyectos de la convocatoria. Estos resultados dan cuenta del trabajo de los actores del proceso de investigación en la zona y de las estrategias implementadas en este trabajo.

\section{¿En qué temas estamos investigando en la ZCBC?}

Dentro de las temáticas más representativas en desarrollo en la última convocatoria del SIUNAD se tiene una participación muy activa en proyectos basados en procesos de argumentación y relacionados con procesos de aprendizaje en nutrición animal, estrategias para la toma de decisiones en el ámbito académico y regional, apoyo en el sistema de educación permanente, procesos de conformación de una red de investigación y estrategias de trabajo colaborativo.

Desde otras miradas se vienen desarrollando proyectos que aportan al desarrollo de las escuelas, el fortalecimiento de los procesos académicos teniendo en cuenta: los egresados de la Universidad, el trabajo con personas en situaciones vulnerables, la implementación de estrategias de aprendizaje desde el uso pedagógico de la visibilidad, el desarrollo de materiales pedagógicos en el marco del conocimiento ancestral y los procesos de diagnóstico psicosocial en jóvenes de regiones de la zona.

Los proyectos de los semilleros de investigación se vienen desarrollando desde estados del arte, proyectos orientados desde la zootecnia, tratamientos especializados de cultivos, un acercamiento a la línea de tecnología con la propuesta de laboratorios remotos desde la ingeniería y se habilita la participación en la red de salud humana, red propuesta y liderada desde la zona.

\section{Visibilidad de los investigadores}

La participación de la ZCBC en el IV Encuentro Nacional de Investigación, realizado en mayo de 2009, fue muy representativa. Se presentaron 17 ponencias, que evidencian los avances y resultados de los proyectos desarrollados desde los semilleros y grupos de investigación. Una 
revisión de estos trabajos permite analizar las tendencias temáticas y las líneas hacia las que apunta la zona.

Una tendencia muy clara es la de la investigación educativa, en donde la Consejería Académica juega un papel importante en la reflexión sobre la práctica de la educación a distancia, las estrategias para el acompañamiento de estudiantes, así como el desarrollo de competencias y hábitos de estudio. Así mismo, existe un interés por el tema de la innovación educativa: el uso de las tecnologías informáticas y digitales para la inclusión social y el desarrollo de simuladores y aulas inteligentes para el aprendizaje autónomo. Otros temas expuestos en estas ponencias en el campo de educación son: la visibilidad desde un enfoque pedagógico y educativo, y el análisis de redes sociales y académicas.

Lo anterior refleja a la Universidad Nacional Abierta y a Distancia como un sistema reflexivo, que a través de la investigación y la innovación tecnológica busca mejorar constantemente su práctica, acorde con las necesidades locales y globales. En ese sentido, se evidencia un importante aporte de la zCBC, al cumplimiento de la visión institucional de posicionar la Universidad como una organización líder en educación abierta y a distancia.

\section{¡Una zona con proyección!}

La ZCBC se favorece cada vez más en sus procesos investigativos, la participación de los actores desde los centros se vine evidenciando en las propuestas de investigación que se adelantan desde las necesidades locales de cada región.

Las estrategias de liderazgo en cada uno de los centros han demostrado que actores de cada centro se apropian del proceso y motivan a sus pares a vincularse con los proyectos de investigación.

La comunicación entre líderes de investigación aporta al trabajo en equipo fortaleciendo los procesos y generando colaboración en las acciones de orientación desde los centros.

El desarrollar actividades en cada una de los centros genera un efecto de descentralización y permite que no solo el centro más grande de la zona (CEAD José Acevedo y Gómez) adquiera mayor visibilidad y por ende mayor reconocimiento, sino que por el contrario cada centro se haga visible y reconocido por la comunidad académica. 